\title{
Observation of thundercloud-related gamma rays and neutrons in Tibet
}

\author{
H. Tsuchiya, ${ }^{1, *}$ K. Hibino, ${ }^{2}$ K. Kawata, ${ }^{3}$ N. Hotta, ${ }^{5}$ N. Tateyama, ${ }^{2}$ M. Ohnishi, ${ }^{3}$ M. Takita, ${ }^{3}$ D. Chen, ${ }^{4}$ J. Huang, ${ }^{6}$ \\ M. Miyasaka, ${ }^{7}$ I. Kondo, ${ }^{1}$ E. Takahashi, ${ }^{1}$ S. Shimoda, ${ }^{1}$ Y. Yamada, ${ }^{1}$ H. Lu, ${ }^{6}$ J. L. Zhang, ${ }^{6}$ X. X. Yu, ${ }^{6}$ Y. H. Tan, ${ }^{6}$ \\ S. M. Nie, ${ }^{8}$ K. Munakata, ${ }^{9}$ T. Enoto, ${ }^{1,10}$ and K. Makishima ${ }^{11}$ \\ ${ }^{1}$ High-Energy Astrophysics Laboratory, Riken, 2-1, Hirosawa, Wako, Saitama 351-0198, Japan \\ ${ }^{2}$ Faculty of Engineering, Kanagawa University, Yokohama 221-8686, Japan \\ ${ }^{3}$ Institute for Cosmic Ray Research, University of Tokyo, Kashiwa 277-8582, Japan \\ ${ }^{4}$ National Astronomical Observatories, Chinese Academy of Sciences, $20 A$ Datun Road, Chaoyang District, 100012 Beijing, China \\ ${ }^{5}$ Faculty of Education, Utsunomiya University, Utsunomiya 321-8505, Japan \\ ${ }^{6}$ Key Laboratory of Particle Astrophysics, Institute of High Energy Physics, Chinese Academy of Sciences, Beijing 100049, China \\ ${ }^{7}$ Caltech, Pasadena, California 91125, USA \\ ${ }^{8}$ Guizhou University for Nationalities, Guiyang 550025, China \\ ${ }^{9}$ Department of Physics, Shinshu University, Matsumoto 390-8621, Japan \\ ${ }^{10}$ Kavli Institute for Particle Astrophysics and Cosmology, Department of Physics and SLAC National Accelerator Laboratory, \\ Stanford University, Stanford, California 94305, USA \\ ${ }^{11}$ Department of Physics, University of Tokyo, 7-3-1, Hongo, Bunkyo-ku, Tokyo 113-0033, Japan
}

(Received 1 July 2011; published 10 May 2012)

\begin{abstract}
During the 2010 rainy season in Yangbajing (4300 $\mathrm{m}$ above sea level) in Tibet, China, a long-duration count enhancement associated with thunderclouds was detected by a solar-neutron telescope and neutron monitors installed at the Yangbajing Comic Ray Observatory. The event, lasting for $\sim 40$ min, was observed on July 22, 2010. The solar-neutron telescope detected significant $\gamma$-ray signals with energies $>40 \mathrm{MeV}$ in the event. Such a prolonged high-energy event has never been observed in association with thunderclouds, clearly suggesting that electron acceleration lasts for $40 \mathrm{~min}$ in thunderclouds. In addition, Monte Carlo simulations showed that $>10 \mathrm{MeV} \gamma$ rays largely contribute to the neutron monitor signals, while $>1 \mathrm{keV}$ neutrons produced via a photonuclear reaction contribute relatively less to the signals. This result suggests that enhancements of neutron monitors during thunderstorms are not necessarily clear evidence for neutron production, as previously thought.
\end{abstract}

DOI: 10.1103/PhysRevD.85.092006

\section{INTRODUCTION}

Recent observations have shown that thunderclouds are powerful particle accelerators, emitting bremsstrahlung $\gamma$ rays that extend to $10 \mathrm{MeV}$ or higher [1-7]. Unlike terrestrial gamma-ray flashes (TGFs) and lightning-related $\gamma$ rays, which last for milliseconds or less, thundercloudrelated $\gamma$ rays are characterized by durations of a few tens of seconds to a few minutes, or occasionally more than $10 \mathrm{~min}$. These thundercloud-related $\gamma$ rays have been thought to be produced by relativistic electrons, in accordance with the relativistic runaway electron avalanche (RREA) model [8-10] that involves acceleration and multiplication of ambient electrons. However, mainly because of the lack of a large sample of thundercloud-related $\gamma$ rays, there is still no consensus that all of those $\gamma$ rays are really generated by the RREA mechanism. It is also unclear whether the charging mechanism of thunderclouds is related to the production of thundercloud-related $\gamma$ rays.

Several groups conducting their experiments on high mountains have reported detecting various particles besides $\gamma$ rays, in possible association with thunderstorms

\footnotetext{
*Japan Atomic Energy Agency, Tokai-mura, Naka, Ibaraki 319-1195, Japan.
}

PACS numbers: 92.60.Pw, 52.38.Ph, 82.33.Xj, 93.30.Db

$[11,12]$. Among such particles, the production of neutrons in coincidence with natural lightning by a thermonuclear reaction ${ }^{2} \mathrm{H}+{ }^{2} \mathrm{H} \rightarrow n(2.45 \mathrm{MeV})+{ }^{3} \mathrm{He}$ was closely investigated in the 1970s-1980s because such neutrons provide a key not only to elucidate the mechanism of lightning but also to know if such neutrons are another source captured by ${ }^{14} \mathrm{C}$. Investigating such neutrons, Shah et al. [13] and Shyam and Kaushik [14] reported detections of $10^{7}-10^{10}$ neutrons per lightning strike.

Instead of the above fusion mechanism, Babich and Roussel-Dupré [15] proposed a photonuclear reaction, ${ }^{14} \mathrm{~N}(\gamma, n){ }^{13} \mathrm{~N}$, showing that the fusion mechanism is not feasible under the usual physical conditions in lightning. The photonuclear reaction begins at a $\gamma$-ray energy of $10.5 \mathrm{MeV}$ [16], and hence may occur because $\gamma$ rays with energies above the threshold have been actually observed. Therefore, photonuclear neutrons provide another clue to solve the nonthermal mechanism in thunderstorms. Actually, Carlson et al. [17] conducted a close investigation on neutron production in TGFs, predicting that a TGF on average produces $\sim 10^{12}$ neutrons corresponding to a ground-level neutron fluence of $(0.03-1) \times 10^{4} \mathrm{~m}^{-2}$. Similarly, Babich et al. [18] also predicted that neutrons with fluence of $10^{3}-10^{7} \mathrm{~m}^{-2}$ would arrive at ground level when energetic $\gamma$ rays are produced under the RREA 
mechanism. Many neutron monitors, installed at cosmicray stations in the world, could detect such neutron bursts in thunderstorms, if those neutrons actually reached them.

Interestingly, a clear enhancement during thunderstorms was recently detected by neutron monitors installed at Mt. Aragats at an altitude of $3250 \mathrm{~m}$ a.s.l. in Armenia [5,6]. In addition, plastic scintillator-based detectors, arranged close to the neutron monitors, detected long-duration (10-20 $\min ) \gamma$ rays extending to $40-50 \mathrm{MeV}$. Generally, owing to its detection method, a neutron monitor is believed to be very sensitive to nucleons but insensitive to $\gamma$ rays and electrons. Thus, Chilingarian et al. [5] have concluded that the observed increase of the Aragats neutron monitor is attributable to neutrons generated via the photonuclear reaction.

Similar to the Armenian case, clear enhancements were occasionally obtained by some detectors installed at the Yangbajing Cosmic Ray Observatory $\left(30.103^{\circ} \mathrm{N}\right.$, $90.523^{\circ} \mathrm{E}$; , cutoff rigidity $=14 \mathrm{GV}$ ), which is located on a mountain $4.3 \mathrm{~km}$ a.s.l. in Tibet, China. Actually, because two electric-field mills (BOLTEK EFM-100) were installed in February 2010 at the observatory, five large count enhancements were found to be associated with electric-field variations in the rainy season. In this paper we present one prolonged count increase with duration of $40 \mathrm{~min}$, which was obtained by both the Yangbajing neutron monitor (YBJ $\mathrm{NM}$ ) and a solar-neutron telescope (SNT). Utilizing the event, we especially discuss how the observed signals are attributed to $\gamma$ rays and neutrons produced via the photonuclear reaction. Then, we deduce fluxes of $\gamma$ rays and neutrons, and compare them with those obtained from other experiments and Monte Carlo predictions.

\section{EXPERIMENT}

Because of its high altitude (4300 m a.s.1.) and meteorological conditions from May to October, the sky above the Yangbajing Cosmic Ray Observatory is frequently covered with thunderclouds. The observatory has three independent detectors: the Tibet air shower array [19], YBJ NM [20], and the SNT [21]. The air shower array, working successfully since 1990, mainly observes $10^{12}-10^{17} \mathrm{eV}$ primary cosmic rays. On the other hand, YBJ NM and the SNT have been operating since 1998, mainly aiming at detecting $>100 \mathrm{MeV}$ solar-flare neutrons and protons to elucidate the ion-acceleration mechanism in solar flares. YBJ NM and the SNT are placed close to each other in one building.

\section{A. Yangbajing neutron monitor}

YBJ NM consists of 28 NM64-type detectors [22,23] having the largest area of $32 \mathrm{~m}^{2}$ among worldwide neutron monitors. An NM64 neutron monitor is composed of a $\mathrm{BF}_{3}$ counter, which is surrounded by polyethylene $\left[\left(\mathrm{C}_{2} \mathrm{H}_{4}\right)_{n}\right]$ plates of thickness $7.5 \mathrm{~cm}$ and lead blocks with an average thickness of $120 \mathrm{~g} \mathrm{~cm}^{-2}$. The polyethylene plates reflect low-energy nucleons accidentally produced in substances close to the detector, while the lead blocks multiply impinging neutrons via inelastic scattering processes.

Each $\mathrm{BF}_{3}$ counter that contains the $\mathrm{BF}_{3}$ gas with the density of $3 \times 10^{-4} \mathrm{~g} \mathrm{~cm}^{-3}$ has a length of $190.8 \mathrm{~cm}$ and radius of $7.4 \mathrm{~cm}$. The counter can easily detect a thermal neutron via a neutron capture reaction as ${ }^{10} \mathrm{~B}+{ }^{1} \mathrm{n} \rightarrow$ ${ }^{4} \mathrm{He}+{ }^{7} \mathrm{Li}$, because the cross section of the capture reaction increases rapidly as the kinetic energy of the neutron decreases to thermal energy. To efficiently decelerate neutrons to thermal energy by elastic collisions with hydrogen nuclei, each $\mathrm{BF}_{3}$ counter is inserted into an additional polyethylene tube with a thickness of $2 \mathrm{~cm}$. A ${ }^{4} \mathrm{He}$ ion created by a neutron capture reaction produces a large amount of ionization loss by $\sim 1 \mathrm{MeV}$ or higher in the $\mathrm{BF}_{3}$ counters to provide a sufficiently large signal on its anode. Because of the multiplication and thermalization of the incident neutron, the large signal has no information about the incident energy. However, the signal can be easily distinguished from charged secondary cosmic-ray background events (mainly muons), which provide a small signal of $\sim 9 \mathrm{keV}$. Output signals from individual counters are fed to the data acquisition system, and the event number of individual counters is recorded every second.

It is widely believed that neutron monitors have no sensitivity to electromagnetic components because of the thick lead blocks. However, a photonuclear reaction between $\gamma$ rays and lead nuclei begins at the $\gamma$-ray energy of $7 \mathrm{MeV}$, and peaks at $\sim 13 \mathrm{MeV}$ [16]. Thus, high-energy $\gamma$ rays associated with energies $>7 \mathrm{MeV}$ can produce neutrons via the photonuclear reaction. Accordingly, neutron monitors might capture such photonuclear neutrons produced by thundercloud-related $\gamma$ rays extending to $10 \mathrm{MeV}$ or higher energies.

In order to investigate this possibility, we performed a Monte Carlo simulation based on GEANT4 [24] and derived detection efficiencies of an NM64 neutron monitor, including YBJ NM, for neutrons, $\gamma$ rays, electrons, and positrons in a wide energy range of $1 \mathrm{keV}-1 \mathrm{GeV}$. For this purpose, a geometry of a standard NM64 neutron monitor [23] was constructed, and $10^{5}$ monoenergetic particles for each species were illuminated on the same area as the neutron monitor. In one monoenergetic simulation, an irradiated particle was isotropically injected toward the neutron monitor from the vertical direction to 60 degrees. We choose in each simulation (including air propagation simulations described later) a hadronic model of QGSP_BERT_HP provided by GEANT4 to treat physics processes of neutrons in the atmosphere.

Figure 1 shows detection efficiencies determined in this manner for the four particles. The present efficiency for neutrons (black circles) agrees well with that obtained by another detector simulation conducted by Clem and Dorman [25] (dashed lines). The difference in efficiencies at $10 \mathrm{MeV}-1 \mathrm{GeV}$ of the two simulations is a maximum 


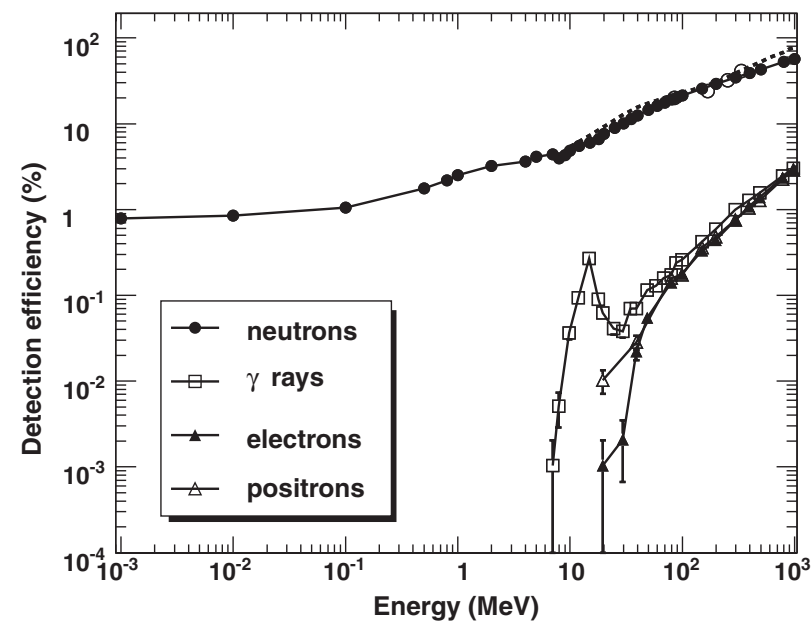

FIG. 1. Detection efficiencies of an NM64 neutron monitor for neutrons, $\gamma$ rays, electrons, and positrons, as determined by the GEANT4 simulation. A dashed curve indicates detection efficiency of an NM64 neutron monitor obtained by Clem and Dorman [25]. Open circles represent experimental results by Shibata et al. [26]. The horizontal axis denotes incident energy in $\mathrm{MeV}$. Errors are statistical $1 \sigma$ errors

$30 \%$. In addition, our results for neutrons can reproduce well efficiencies experimentally determined using an accelerator neutron beam [26]. These consistencies validate our simulation results.

As expected, the present simulation reveals that an NM64 neutron monitor has sensitivity to electromagnetic components in energies ranging from $10 \mathrm{MeV}$ to $1 \mathrm{GeV}$. Compared with the efficiencies for neutrons, those for $\gamma$ rays in the energy range are lower by a factor of $1 / 125-1 / 20$. Similarly, high-energy electrons and positrons entering the lead blocks emit $\gamma$ rays via bremsstrahlung, which in turn generate either neutrons via the photonuclear process or electrons via pair creation. Since the critical energy of electrons in lead is $7 \mathrm{MeV}$, these cascading processes would continue until energies of electrons and $\gamma$ rays are below the critical energy. As the incident energy of electromagnetic components increases, the cascading becomes more effective in causing the photonuclear reaction. Thus, detection efficiencies for electromagnetic components increase (Fig. 1).

\section{B. Tibet solar-neutron telescope}

Here, we provide minimal information necessary to understand events reported in this paper; detailed information on the Tibet SNT, including detection efficiencies for neutrons and $\gamma$ rays, is presented in Muraki et al. [21].

The SNT installed at the observatory is part of the international solar-neutron observation network. It is composed of nine plastic scintillation counters and proportional counters that are placed around them. A plastic scintillation counter contains plastic scintillator blocks of area and thickness of $1 \mathrm{~m}^{2}$ and $40 \mathrm{~cm}$, respectively. Thus, the total area of the plastic scintillators is $9 \mathrm{~m}^{2}$. The counter has a $\phi 12.7 \mathrm{~cm}$ photomultiplier at the top of the counter for collecting light emissions originating from incident particles.

Incident charged particles deposit their energies in the thick plastic scintillators via ionization loss, and hence can be readily observed with the SNT. Incident neutrons produce recoil ions by scattering protons or carbons in the plastic scintillators, while $\gamma$ rays produce electrons via Compton scattering or pair creation. Through these processes, the SNT is able to measure neutrons and $\gamma$ rays, although it does not differentiate between them. In addition, output signals from the photomultiplier are fed to the data acquisition system, amplified, and discriminated at 4 levels, which correspond to energy deposits of an incident particle of $>40,>80,>120$, and $>160 \mathrm{MeV}$. For each of the nine plastic scintillation counters, an individual discriminated logical signal is counted by scalers every second.

Proportional counters complement the plastic scintillation counters. A proportional counter has a length of $330 \mathrm{~cm}$ and radius of $5 \mathrm{~cm}$, and contains $90 \% \mathrm{Ar}$ and $10 \% \mathrm{CH}_{4}$. Thirty proportional counters are placed above the nine plastic scintillation counters, while 72 proportional ones shield the 4 sides of the plastic scintillation ones. Therefore, the surrounding counters can be utilized as an anticounter to separate photons and neutrons from charged particles. In fact, using the surrounding proportional counter signals in anticoincidence, the four discriminated counting rates of the central plastic counters are reduced by a factor of $0.2-0.25$

\section{Electric-field mill}

To measure electric-field variations, two commercial electric-field mills (BOLTEK EFM-100) were installed on the premises. One is mounted on the ground, while the other is located on the roof of a central building; hereafter, denoted as EFM1 and EFM2, respectively. The two mills are arranged $\sim 25 \mathrm{~m}$ apart with a vertical distance of $3.4 \mathrm{~m}$. Individual output signals are transmitted to the central building with optical cables, directly fed to PCs, and recorded every $0.1 \mathrm{~s}$ as the electric-field strength in the range $\pm 40 \mathrm{kV} \mathrm{m}^{-1}$ with a resolution of $20 \mathrm{~V} \mathrm{~m}^{-1}$.

The electric-field strength measured by EFM2 is always higher, by a factor of $\sim 2$, than that by EFM1. Such an enhancement of an electric field is often caused by distortion of local electric-field lines because of obstructions such as a building. In fact, EFM2 is installed near a corner of the roof of a building, and hence is more largely affected by such distortion than EFM1, which is located on the ground with few surrounding obstructions. Considering this disparity, we use only EFM1 data in this paper.

\section{OBSERVATIONS}

\section{A. Overview}

Examining the data over the 2010 rainy season from May to October, we visually found 25 events in which 
electric fields largely deviate from fair-weather states of $<100 \mathrm{~V} \mathrm{~m}^{-1}$. Five of them accompanied prolonged count enhancements. Three of the five events are clearly observed by either YBJ NM or the SNT, lasting for 10-20 min. Similar events with duration of 10-20 min have been already reported by other measurements (for example, [3,5]). On the other hand, the remaining two events, detected by both YBJ NM and the SNT, last for $>30 \mathrm{~min}$. Such a long-lasting emission has never been observed.

For two reasons, we selected one of the two events that are detected by both YBJ NM and the SNT. One is that the event is a fast observation of the longest-duration emission among other long-duration events. The other is that the selected event clearly correlates with electric fields (as shown later), while the other has only a poor correlation with electric fields. Although the statistical significance of the latter event for YBJ NM and the SNT ( $>40 \mathrm{MeV}$ ) was around 2 times higher than the selected event, we will have to collect additional ones in order to understand the nature of such a poorly correlated event.

\section{B. Count histories}

Figure 2 shows 5 min counting rates by YBJ NM and the SNT and $1 \mathrm{~s}$ electric-field variations obtained over 3:007:00 UT on July 22, 2010. All counting rates by YBJ NM and the SNT are corrected for atmospheric pressure variations. In this event, YBJ NM count rates and the SNT ones in $>40 \mathrm{MeV}$ clearly increase around 5 UT [Figs. 2(a) and 2(b)]. In addition, higher-energy channels of the SNT [Figs. 2(b)-2(e)] appear to show count enhancements in coincidence with the above clear increases. Given the clear signals, in particular, the $>40 \mathrm{MeV}$ channel of the SNT vetoing charged particles with the anticounter [Fig. 2(b)], we can conclude that $>40 \mathrm{MeV} \gamma$ rays and/or neutrons reach the detectors to produce the observed signals.

With a criterion that individual counts of the $>40 \mathrm{MeV}$ channel of the SNT continuously have $>2 \sigma$ statistical significance above background, we define burst time as $40 \mathrm{~min}$ at 4:30-5:10 UT. Here, by excluding the data in this period and fitting the remaining data with a quadratic function, we estimate the background for YBJ NM and the SNT (gray dashed curves in Fig. 2). Subtracting the interpolated background from total observed counts in the burst period, we obtain net count increases for the burst recorded by YBJ NM and the SNT; these are listed in Table I together with their statistical significance. Hereafter, the burst is simply called 100722 .

Generally, the counting rate of a neutron monitor, including YBJ NM, does not simply obey Poisson distribution because of the multiplication of one incident neutron in the lead blocks. Usoskin et al. [27] provide a more detailed explanation on how these effects cause nonPoissonian fluctuations in NM data. They described that a statistical significance obtained by a NM usually should be reduced by a factor of 1.2-2, depending on the geomagnetic cut-off rigidity and the atmospheric depth at NM locations. Thus, the statistical significance obtained (Table I) may decrease by half. Importantly, both YBJ NM and the SNT simultaneously recorded large enhancements in association with electric-field variations.

Based on the following features of the event observed, we may conclude that it is associated with thunderclouds, but not lightning. First is its long duration; apparently, such a long-duration emission would not be generated by lightning and/or its related phenomena that generally last for milliseconds or less. Second is that the electric-field strength in the burst period does not change rapidly (within $1 \mathrm{~s}$ ), but gradually [Fig. 2(f)]. In addition, although not homogeneous, these features have already been reported by many groups $[1-5,7,11,28]$ as thundercloud-related emissions.

\section{Relation with electric fields}

Figure 3 represents detailed time variations of YBJ NM and the SNT, together with the averaged electric fields. Clearly, peaks of YBJ NM and the SNT signals for 100 722, obtained over 4:50-5:04 UT, correlate with those of electric fields in the same interval [Figs. 3(a) and 3(b)]. For clarity, Fig. 4 shows the correlation between the present burst and the electric field measured by EFM1. We computed a correlation coefficient between the count variations of YBJ NM and the SNT and the electric field as 0.79 (0.01) and $0.77(0.03)$, respectively. Each number in parentheses represents a correlation coefficient outside the burst period.

An electric field in the downward direction is measured as a positive field. Thus, the positive electric fields correspond to the existence of positive charges overhead, which are frequently observed at Tibet [29] and New Mexico [30] when thunderclouds exist at a mature stage over a field mill on the ground. Furthermore, such a thundercloud generally forms tripole electrical structures, which consist of positive, negative, and positive layers from top to bottom, which in turn accelerate electrons therein toward the ground.

\section{NEUTRON PRODUCTION AND PROPAGATION IN THE AIR}

\section{A. Outline}

According to Babich et al. [31], a yield rate of a photonuclear neutron per one gamma ray with energies $>10 \mathrm{MeV}$ is $4.3 \times 10^{-3}$. Produced neutrons propagate in the atmosphere, attenuated by elastic and/or inelastic collisions with air nulcei. Assuming neutrons propagate over $L=1 \mathrm{~km}(0.1 \mathrm{~km})$ to reach the observatory, neutrons produced decrease in number by a factor of $\exp \left(-L / \lambda_{\mathrm{n}}\right)=$ $2 \times 10^{-3}(0.5)$. Here, $\lambda_{\mathrm{n}}$ represents an attenuation length of neutrons in the atmosphere, calculated as $\lambda_{\mathrm{n}}=13 \mathrm{~g} \mathrm{~cm}^{-2}$ 


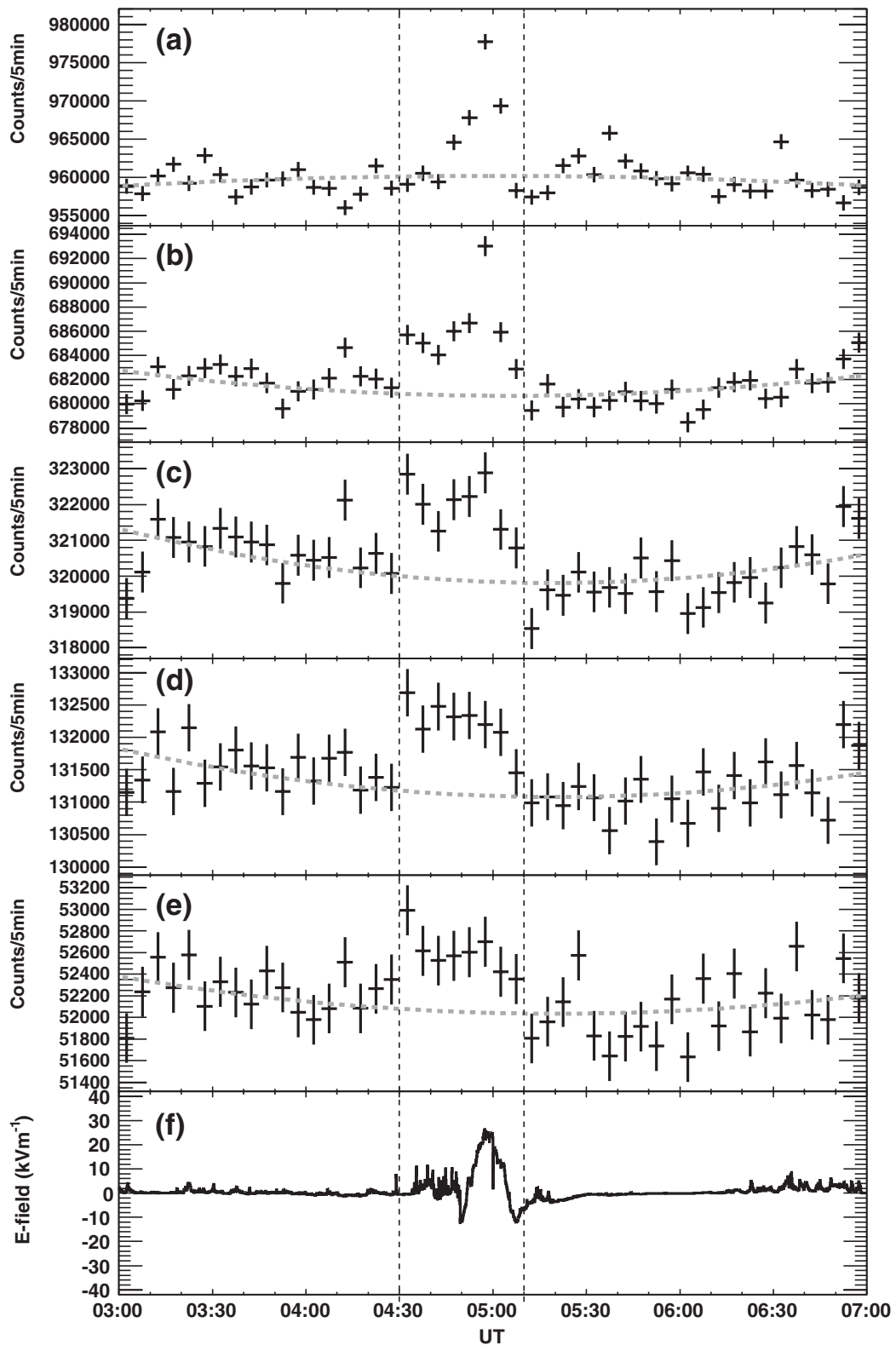

FIG. 2. Count rates per 5 min detected by YBJ NM and the SNT, and $1 \mathrm{~s}$ variations of EFM1 over 3:00-7:00 UT on July 22, 2010. Panel (a) shows count rates by YBJ NM; while panels (b)-(e) show count rates by $>40 \mathrm{MeV},>80 \mathrm{MeV},>120 \mathrm{MeV}$, and $>160 \mathrm{MeV}$ SNT with anticoincidence. Panel (f) shows the $1 \mathrm{~s}$ variations by EFM1. Dashed gray curves in panels (a)-(e) indicate the estimated background, while vertical dashed lines in all panels represent the defined burst periods. The horizontal axes show universal time. Error bars are statistical $1 \sigma$ except for panel (f).

TABLE I. Net count increases and statistical significance.

\begin{tabular}{lc}
\hline \hline & $\Delta \mathrm{N}^{\mathrm{a}}$ (significance) \\
\hline YBJ NM & $34000 \pm 4200(8.1 \sigma)$ \\
$\mathrm{SNT}>40 \mathrm{MeV}$ & $44000 \pm 3500(13 \sigma)$ \\
$\mathrm{SNT}>80 \mathrm{MeV}$ & $16000 \pm 2400(6.7 \sigma)$ \\
$\mathrm{SNT}>120 \mathrm{MeV}$ & $8700 \pm 1500(5.8 \sigma)$ \\
$\mathrm{SNT}>160 \mathrm{MeV}$ & $4600 \pm 970(4.7 \sigma)$ \\
\hline \hline
\end{tabular}

${ }^{a}$ Each quoted error includes fluctuations of the background and total observed counts. for $20 \mathrm{MeV}$ neutrons using a total cross section between a neutron and an air nucleus [32]. As a result, a $>10 \mathrm{MeV} \gamma$ ray is found to produce $10^{-5}-10^{-3}$ neutrons to arrive at the observatory. Given this arrival rate of neutrons and derived detections efficiencies for neutrons and $\gamma$ rays (Fig. 1), we expect that $>10 \mathrm{MeV} \gamma$ rays would be able to considerably contribute to the signals detected by YBJ NM. To better understand how much photonuclear neutrons propagate to the observatory, we performed a GEANT4 simulation.

For the purpose of simulating neutron production via the photonuclear reaction and neutron propagation in the 


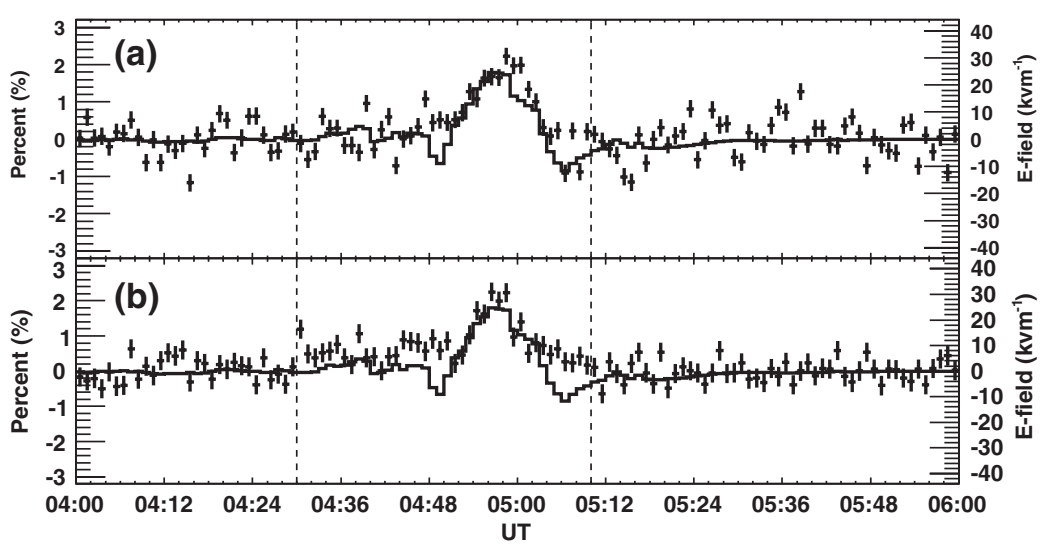

FIG. 3. One-minute count histories in percent observed by YBJ NM and the SNT, and 1 min averaged electric-field variations. Data points with $1 \sigma$ error bars in panels (a) and (b) correspond to the variations in YBJ NM and $>40 \mathrm{MeV}$ SNT with anticoincidence for 100722 , respectively. In all panels, histograms (solid lines) represent the average field variations by EFM1. The horizontal axes show universal time. Left and right vertical axes denote the count variations in percent and the electric-field strength in $\mathrm{kVm}^{-1}$, respectively. Vertical lines in each panel indicate the burst onset and end times.

atmosphere, we constructed five atmospheric layers starting from the observatory level $(4.3 \mathrm{~km}$ a.s.1.) to $5 \mathrm{~km}$ higher. Each rectangular atmospheric layer has a vertical length ( $z$ direction) of $1 \mathrm{~km}$ and horizontal length ( $x y$ directions) of $10 \mathrm{~km}$, and consists of $\mathrm{N}_{2}, \mathrm{O}_{2}$, and $\mathrm{Ar}$ with mole ratios of $78.1 \%, 21.0 \%$, and $0.9 \%$, respectively. Air density in the individual layers is fixed at $7.7 \times 10^{-4} \mathrm{~g} \mathrm{~cm}^{-3}$ for $4.3-5.3 \mathrm{~km}$ a.s.1., $7.0 \times 10^{-4} \mathrm{~g} \mathrm{~cm}^{-3}$ for $5.3-6.3 \mathrm{~km}$ a.s.1., $6.6 \times 10^{-4} \mathrm{~g} \mathrm{~cm}^{-3}$ for $6.3-7.3 \mathrm{~km}$ a.s.1., $\quad 5.6 \times 10^{-4} \mathrm{~g} \mathrm{~cm}^{-3}$ for $7.3-8.3 \mathrm{~km} \mathrm{a.s.1.,} \mathrm{and}$ $5.0 \times 10^{-4} \mathrm{~g} \mathrm{~cm}^{-3}$ for $8.3-9.3 \mathrm{~km}$ a.s.1 [33]. In the following simulations, seven source heights are assumed to be $0.3,0.6,0.9,1.5,2,3$, and $5 \mathrm{~km}$ above the observatory level. From each source height, $1 \times 10^{6} \gamma$ rays were injected to the atmosphere to produce secondary particles. The secondary particles, propagating to the observatory, were saved with their species, energy, $x-y$ positions, azimuth, and zenith angles.

Bremsstrahlung $\gamma$ rays derived from runaway electrons have been thought to have an exponentially cut-off powerlaw spectrum, with a cut-off energy of $\sim 7 \mathrm{MeV}[34,35]$.

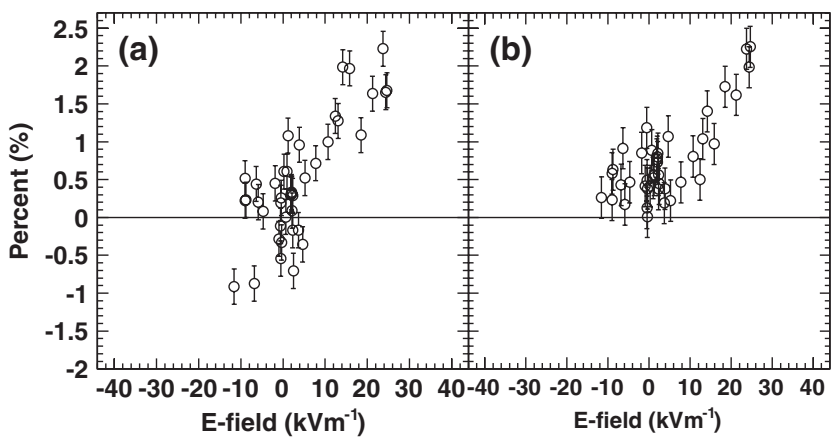

FIG. 4. Burst count variations plotted against electric fields measured by EFM1. Open circles are count variations in the burst intervals shown in Fig. 3. Panels (a) and (b) correspond to YBJ NM and the SNT ( $>40 \mathrm{MeV}$ ) for 100722 , respectively.
However, the recent AGILE observation [36] indicated that a high-energy part $(>1 \mathrm{MeV})$ of the TGF spectrum extending from 10 to $100 \mathrm{MeV}$ can be explained by a power-law spectrum with a spectrum index, $\beta$, of -2.7 rather than an exponentially cut-off one. Sea-level observations of longduration $\gamma$ rays also showed that a source $\gamma$-ray spectrum may be described as a power-law type with $\beta \sim-2$ [7]. Theoretically, $\beta$ of a bremsstrahlung $\gamma$-ray spectrum has the hardest limit of -1 . We therefore assumed a power-law spectrum as an initial photon spectrum in this study and $\beta$ is $-1,-2$, or -3 . The minimum and maximum energies of the spectrum are set at 10 and $300 \mathrm{MeV}$, respectively, to fully cover the presently relevant energy range. In addition, downward directions of initial $\gamma$ rays were assumed to be distributed either isotropically within 0-30 degrees or over a Gaussian beam with a half-opening angle of 30 degrees. Both types would be expected from runaway electrons moving in electric fields in air, because moving electrons are subjected to multiple scatterings with air molecules, and the geometrical or electrical structure of electric fields in thunderclouds may not be very simple [37].

\section{B. Energy spectrum}

Figures 5 and 6 show neutron energy spectra obtained by the isotropic and Gaussian angular distributions, respectively. There is no significant difference in shape of the neutron spectra between the two angular distributions. These neutron spectra suggest that the neutrons arriving at the observatory have a mean energy of $1-10 \mathrm{MeV}$ and the maximum energy of produced neutrons is about onethird of that of the $\gamma$ rays emitted from a source. The former feature has been reported by Carlson et al. [17] and Babich et al. [31] as well.

Figure 7 represents spectra for $\gamma$ rays and electrons assuming the isotropic emission of initial $\gamma$ rays. Similar to neutron spectra, those for $\gamma$ rays and electrons, assuming 

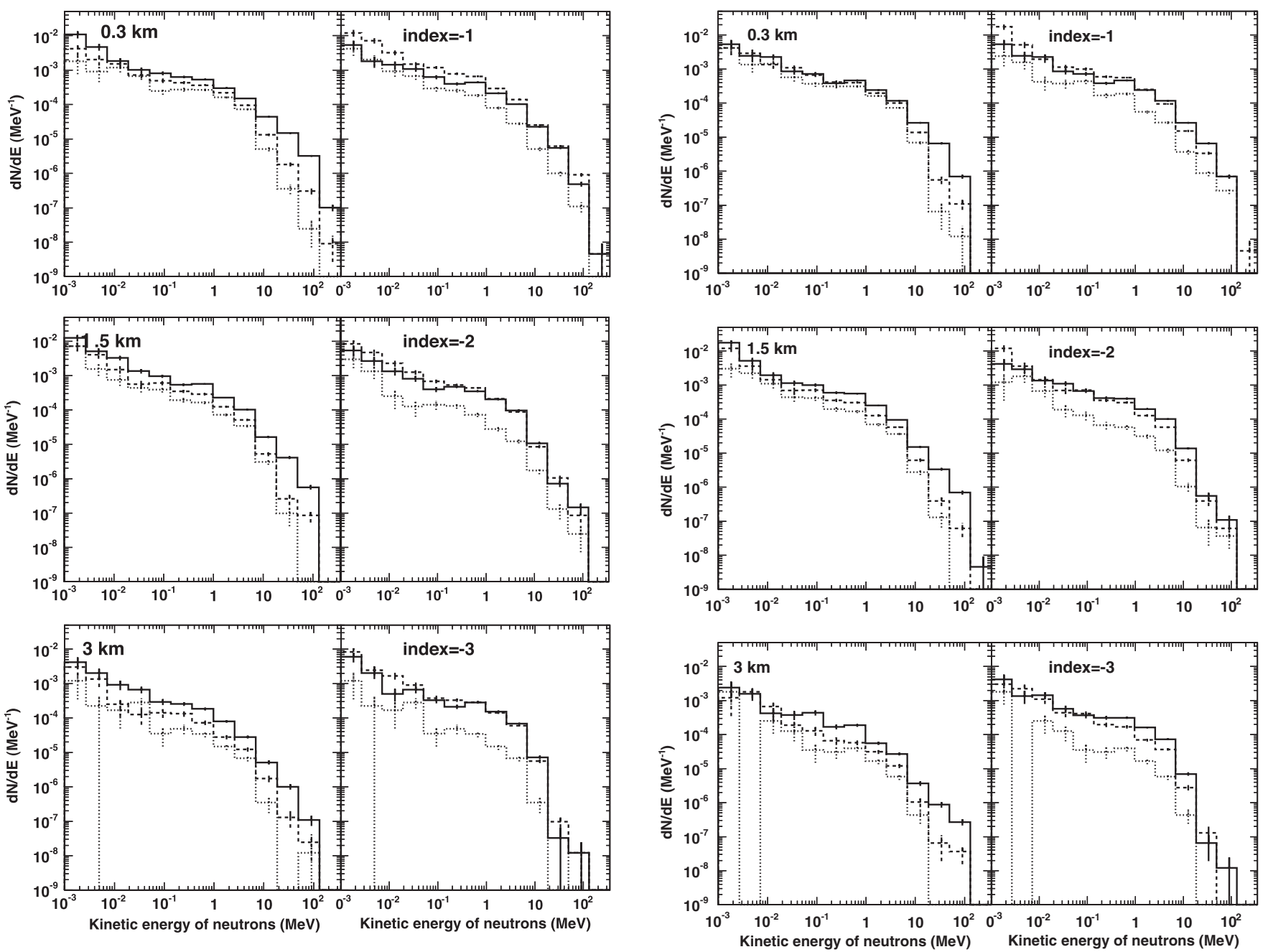

FIG. 5. Derived energy spectra of neutrons reaching the observatory level, assuming the isotropic emission of initial $\gamma$ rays. Left panels show those spectra with an initial source height fixed at a given value in each panel. Solid, dashed, and dotted lines in the left panels correspond to $\beta$ of $-1,-2$, and -3 , respectively. Right panels indicate those spectra in which $\beta$ is fixed at a constant value with source heights of 0.3 (solid), 1.5 (dashed), and $3 \mathrm{~km}$ (dotted). Each horizontal axis denotes kinetic energy of neutrons in $\mathrm{MeV}$ at the observatory. The vertical axes indicate relative values divided by the number of incident $\gamma$ rays $1 \times 10^{6}$.

the Gaussian beam emission, do not largely change from the isotropic ones.

\section{Survival rate}

Figure 8 shows survival rates for $>1 \mathrm{keV}$ neutrons and $>10 \mathrm{MeV} \gamma$ rays for the two angular distributions, sampling arriving neutrons ( $\gamma$ rays) with energies of $>1 \mathrm{keV}$ $(>10 \mathrm{MeV})$ and calculating a ratio of the number of the arriving neutrons ( $\gamma$ rays) to that of primary $\gamma$ rays. The threshold energy of $1 \mathrm{keV}$ for neutrons does not affect our results, because neutrons with energies $<1 \mathrm{keV}$ constitute a maximum $5 \%$ of all neutrons produced. As expected, the neutron survival rates for the two angular distributions are

FIG. 6. Same as Fig. 5, but for Gaussian-type angular distribution of initial $\gamma$ rays.

similar in shape and intensity, having at most $10 \%$ difference in rate. Depending on spectrum indices, the neutron survival rates are generally constant at $\sim 10^{-3}$ until the source height is around $1 \mathrm{~km}$, and then decrease to $10^{-4}-10^{-5}$. The derived survival rates quite agree with those simply calculated in Sec. IVA.

As can be easily seen, each neutron survival rate has its peak at the source height of $\sim 0.6 \mathrm{~km}$, which corresponds to $\sim 50 \mathrm{~g} \mathrm{~cm}^{-2}$. The shape of the survival rates of neutrons simply reflects the product of the probability that the photonuclear reaction occurs at the point $\gamma$ rays propagate in the atmosphere and that the produced neutrons are attenuated, which is proportional to $\left[1-\exp \left(-H / \lambda_{\mathrm{p}}\right)\right] \times$ $\exp \left(-H / \lambda_{\mathrm{n}}\right)$. Here, $H$ represents the assumed source height, while $\lambda_{\mathrm{p}}$ and $\lambda_{\mathrm{n}}$ represent the interaction length of $\gamma$ rays to cause photonuclear reaction, which is $\sim 3000 \mathrm{~g} \mathrm{~cm}^{-2}$ at the peak cross section of $15 \mathrm{mb}$, and the attenuation length of neutrons, respectively. $\lambda_{\mathrm{n}}$ in the relevant neutron energies of $1-100 \mathrm{MeV}$ range between 20 and $100 \mathrm{~g} \mathrm{~cm}^{-2}$, corresponding to $0.2-1.4 \mathrm{~km}$. 

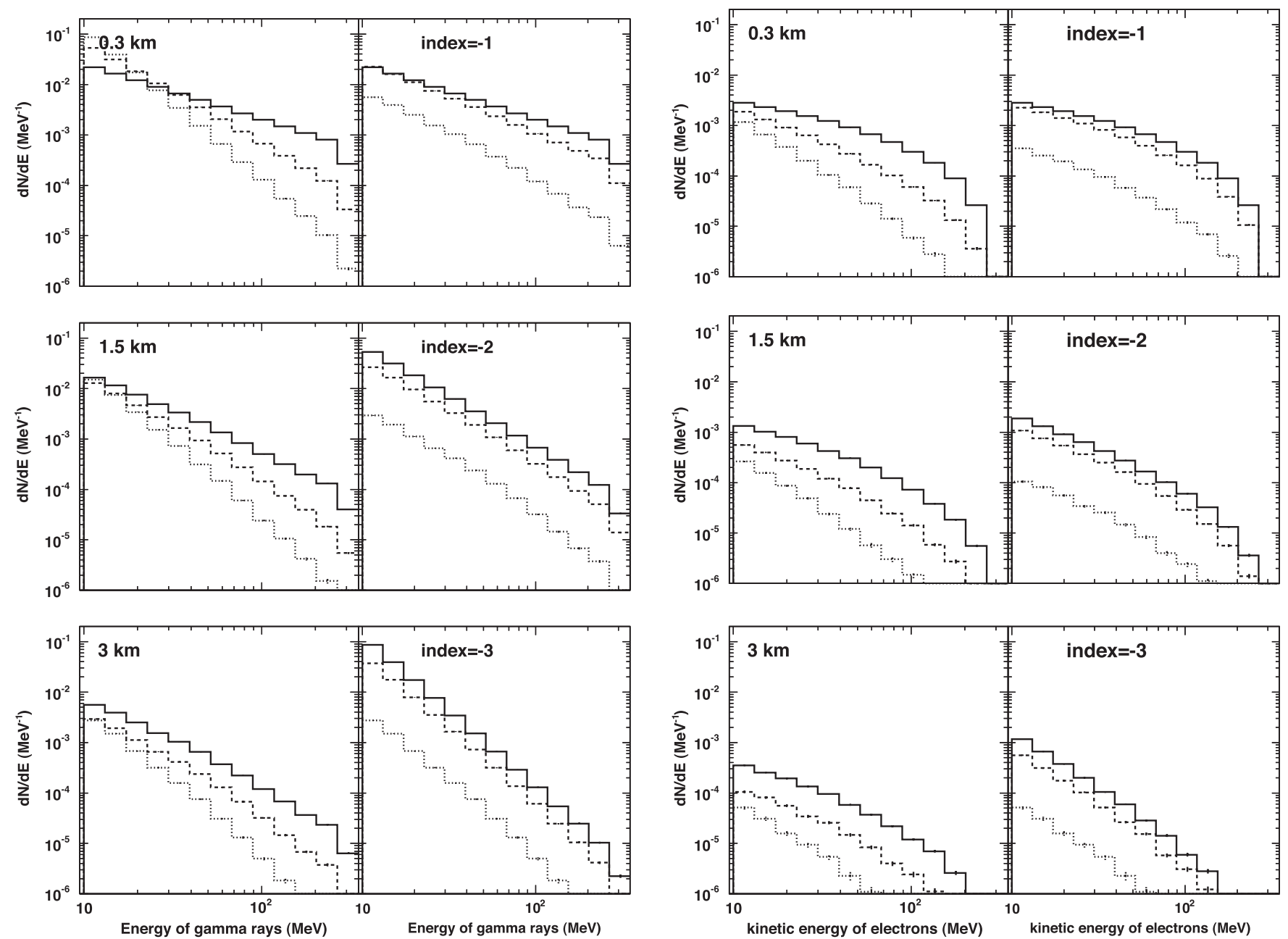

FIG. 7. Same as Fig. 5, but for gamma rays (left) and electrons (right).
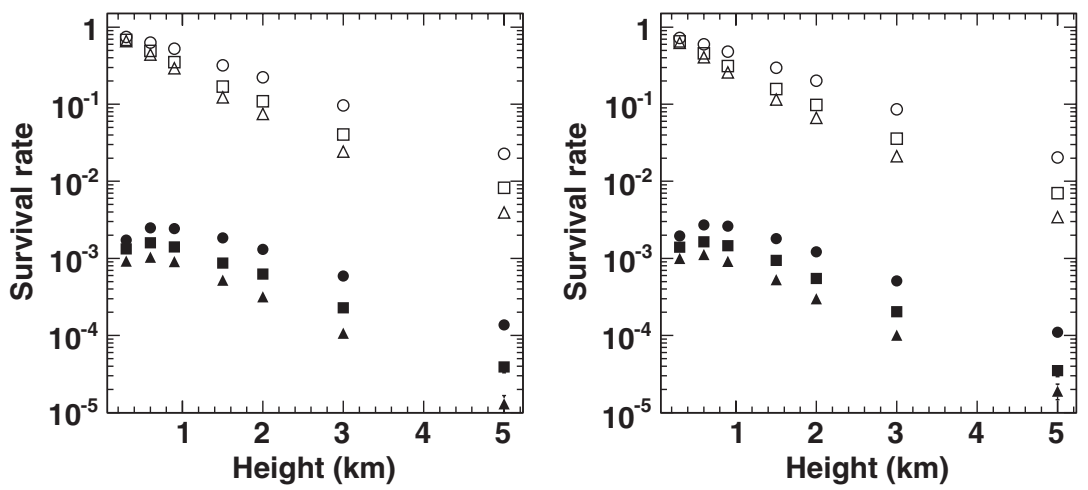

FIG. 8. Survival rates of $>1 \mathrm{keV}$ neutrons (filled) and $>10 \mathrm{MeV} \gamma$ rays (open) at the observatory level, obtained by the $\gamma$-ray power-law spectrum simulations. Left and right panels indicate the isotropic and Gaussian beam distributions of initial $\gamma$ rays, respectively. Circles, squares, and triangles correspond to $\beta$ of $-1,-2$, and -3 , respectively. The horizontal axes show source heights. Errors are statistical $1 \sigma$ errors. 


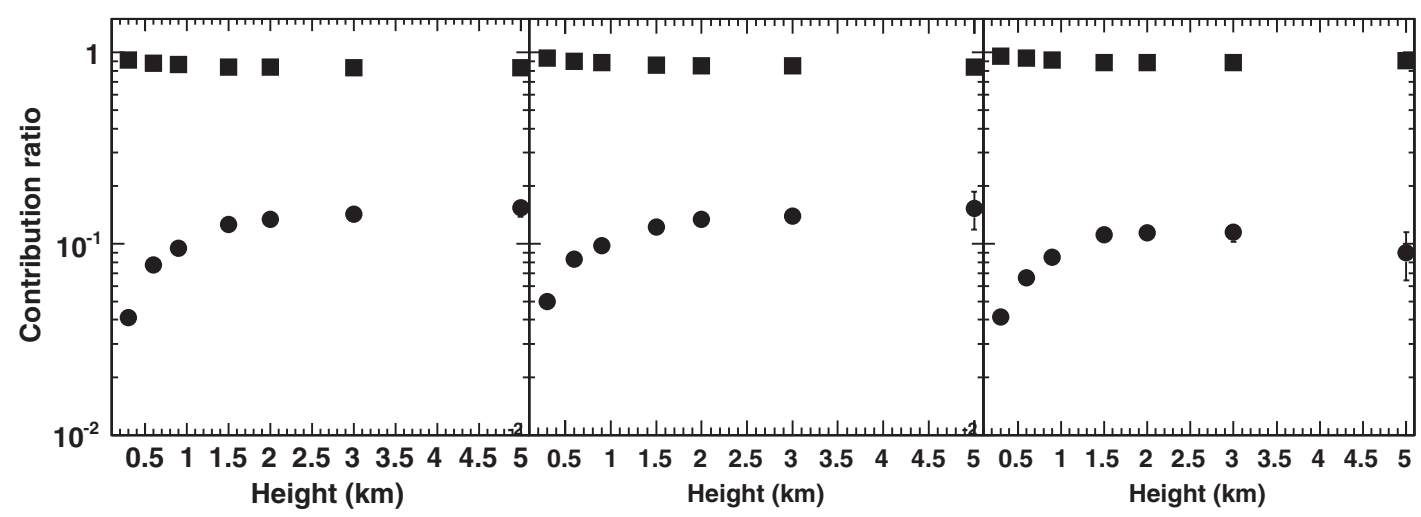

FIG. 9. Contribution ratios of $\gamma$ rays (squares) and neutrons (circles) for YBJ NM signals, plotted against assumed source heights. Left, middle, and right panels correspond to $\beta$ of $-1,-2$, and -3 , respectively. Errors are statistical $1 \sigma$ errors.

\section{CONTRIBUTION RATIOS TO THE SIGNALS}

\section{A. Method}

Given the simulated neutron spectra (Figs. 5 and 6), and those of $\gamma$ rays and electrons (Fig. 7), as well as the detection efficiencies of the neutron monitor (Fig. 1), we can examine how neutrons and electromagnetic components contribute to signals that are expected to be detected by YBJ NM and the SNT during thunderstorms.

As argued so far, we presume that the four components, neutrons, $\gamma$ rays, electrons, and positrons, explain the count increases observed by YBJ NM, and that neutrons and $\gamma$ rays contribute to the SNT signals because the SNT utilizes the anticounter to reject charged particles. Therefore, a predicted count increase at a given time $t$ for individual particles, $\Delta n_{i}(t)$, is written by

$$
\Delta n_{i}(t)=\alpha A \int_{K_{1}}^{K_{2}} I(t) S_{i}\left(E_{i}\right) \epsilon_{i}\left(E_{i}\right) d E_{i}
$$

assuming that the relevant particles have the same production history, $I(t)$, to be generated during thunderstorms. Here, $\alpha$ represents a normalization factor with the unit $\mathrm{MeV}^{-1} \mathrm{~s}^{-1} \mathrm{~m}^{-2}$ for a source spectrum and $A$ represents the area of YBJ NM $\left(32 \mathrm{~m}^{2}\right)$ or the SNT $\left(9 \mathrm{~m}^{2}\right) . E_{i}$ denotes the energy of a particle type $i, S_{i}\left(E_{i}\right)$ represents the spectra (Fig. 5 for isotropic emissions), and $\epsilon_{i}\left(E_{i}\right)$ denotes the detection efficiencies of YBJ NM (Fig. 1) or the SNT (Fig. 5 of [21]). In the present study, $K_{1}$ is set to $1 \mathrm{keV}$ for neutrons and $10 \mathrm{MeV}$ for electromagnetic components, while $K_{2}$ is fixed at $300 \mathrm{MeV}$ for all components. By integrating Eq. (1) over a certain time interval of $t_{2}-t_{1}$, we can obtain an expected net count increase due to each particle $\left(\Delta N_{i}\right)$ as

$$
\Delta N_{i}=\int_{t_{1}}^{t_{2}} \Delta n_{i}(t) d t
$$

Under the present assumption, the simulated spectra $S_{\mathrm{i}}\left(E_{\mathrm{i}}\right)$ in Eq. (1) are independent of time $t$. Accordingly, a ratio of $\Delta N_{i} / \sum \Delta N_{i}$ is calculated as

$$
\frac{\Delta N_{i}}{\sum \Delta N_{i}}=\frac{\int_{K_{1}}^{K_{2}} S_{i}\left(E_{i}\right) \epsilon_{i}\left(E_{i}\right) d E_{i}}{\sum \int_{K_{1}}^{K_{2}} S_{i}\left(E_{i}\right) \epsilon_{i}\left(E_{i}\right) d E_{i}},
$$

which shows the contribution fraction of each species to an expected signal.

\section{B. YBJ NM signals}

Figure 9 depicts contribution ratios of neutrons and $\gamma$ rays for YBJ NM, assuming an isotropic angular distribution. As expected, contribution ratios for the Gaussian angular distribution are almost the same as those for the isotropic distribution. For clarity, contribution ratios for electrons and positrons are not shown. Interestingly, the contribution ratios of neutrons and $\gamma$ rays do not depend largely on $\beta$. Therefore, it is obvious that $\gamma$ rays dominate (96\% to 85\%) the fraction of the expected count increase as the source is farther, while neutrons contribute a maximum of $15 \%$.

\section{SNT signals}

Similarly, contribution ratios for the SNT signals can be calculated using detection efficiencies for neutrons and $\gamma$ rays in Fig. 5 of Muraki et al. [21]. Ninety-nine percent of the observed signal for the $>40 \mathrm{MeV}$ channel of the SNT is dominated by $\gamma$ rays, while the remaining three higherenergy channels are almost fully contributed by $\gamma$ rays. These results for the SNT are mainly ascribed to a relatively small fraction $(<5 \%)$ of neutrons produced in $>40 \mathrm{MeV}$ energies via the photonuclear reaction (Figs. 5 and 6).

\section{TIME HISTORIES OF YBJ NM AND THE SNT}

$I(t)$ can be naturally assumed to follow the time history of the electric field [Fig. 2(f)]. Utilizing the $1 \mathrm{~s}$ electricfield variations as $I(t)$, Eq. (1) has only one unknown parameter, $\alpha$, that needs to be determined by comparing an expected time profile of YBJ NM or the SNT to the observed 1 min profile. Further, we test the following two hypotheses. First, the relevant particles are produced only 

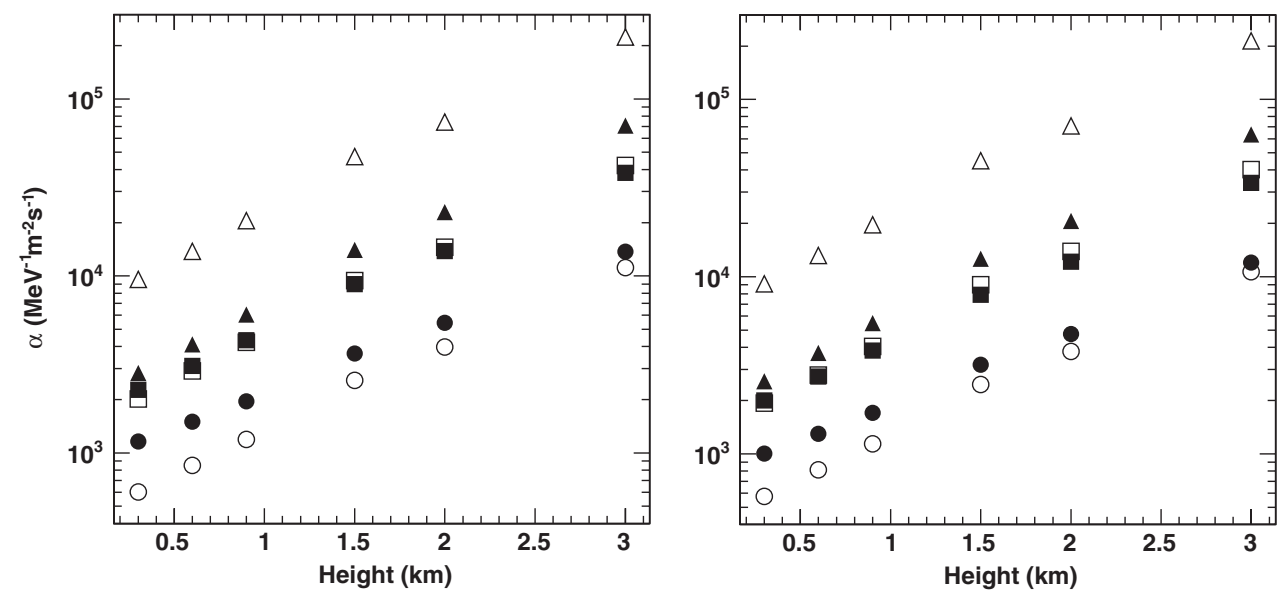

FIG. 10. Comparison of derived $\alpha$ for YBJ NM (open symbols) and the SNT (filled symbols). The left panel shows the negative emission, while the right one denotes the bipolar emission. Circles, squares, and triangles correspond to $\beta$ of $-1,-2$, and -3 , respectively. The horizontal axis in each panel represents assumed source height in $\mathrm{km}$.

when the electric field at the surface has positive polarity and electrons in thunderclouds are accelerated toward the ground. Second, the particles are generated when the field has negative and positive polarities.

For the purpose of introducing the mathematical form of $I(t)$ for the first assumption, the positive electric-field strength [Fig. 2(f)] in the burst periods is divided by the maximum strength of $26.8 \mathrm{kV} \mathrm{m}^{-1}$, and the negative electric-field strength and that outside the individual burst periods are set to zero. On the other hand, absolute values of the electric-field strength divided by the above mentioned maxima are considered as $I(t)$ for the second assumption. In addition, $I(t)$ in this case is zero outside the burst times. Therefore, $\int I(t) d t$ for both assumptions is normalized to one. Hereafter, we call the first and the second assumptions "negative emission" and "bipolar emission," respectively. Substituting each $I(t)$ in Eq. (1) and integrating Eq. (2) every $60 \mathrm{~s}$ in individual burst intervals, we can prepare a 1 min expected time profile depending on each $I(t)$ and compare it with the observed 1 min counting rate of YBJ NM and the SNT (Fig. 3). Next, we compute

$$
\chi^{2}=\sum_{i}\left[\frac{N_{\mathrm{ob}}\left(t_{i}\right)-N_{\mathrm{ex}}\left(t_{i}\right)}{\sigma_{\mathrm{ob}}\left(t_{i}\right)}\right]^{2}
$$

to search for the $\chi^{2}$ minimum with $\alpha$ being a free parameter. Here, $N_{\mathrm{ob}}\left(t_{i}\right)$ and $N_{\mathrm{ex}}\left(t_{i}\right)$ represent the observed $1 \mathrm{~min}$ counts of YBJ NM or the SNT and the model-predicted counts at a given time $t_{i}$ in the burst intervals, respectively. Statistical errors associated with $N_{\mathrm{ob}}\left(t_{i}\right)$ are written as $\sigma_{\mathrm{ob}}\left(t_{i}\right)$. Summation was carried out over each burst interval.

In fact, each $I(t)$ produces the same shape of predicted count history and the same $\chi^{2}$ minimum for YBJ NM or the SNT, despite using simulated spectra $\left[S_{\mathrm{i}}\right.$ in Eq. (1)] obtained with various sets of $\beta$ and $H$. This is because Eq. (1) has only one unknown parameter $\alpha$, and the shape of $S_{\mathrm{i}}$ in Eq. (1) is independent of $t$. To concretely determine $\alpha$, we first independently evaluated $\alpha$ for YBJ NM and the SNT with each $I(t)$ by the above method, using simulated spectra obtained by 21 combinations of $(\beta, H) ; \beta$ of -1 , $-2,-3$ and $H$ of $0.3,0.6,0.9,1.5,2,3$, and $5 \mathrm{~km}$. The derived $\alpha$ for YBJ NM and the SNT are shown in Fig. 10. Next, subtracting $\alpha$ acquired from YBJ NM data with a set of $(\beta, H)$ from that acquired from the SNT data with the same set of $(\beta, H)$, we searched for the smallest difference in $\alpha$ obtained by the two independent detectors.

As clearly seen in Fig. 10, a difference in $\alpha$ for YBJ NM and the SNT is the smallest at $(\beta, H)=(-2,900 \mathrm{~m})$ and $(-2,600 \mathrm{~m})$ for the negative emission and bipolar emission, respectively. Table II displays the calculated $\alpha$ and $\chi^{2}$

TABLE II. $\quad \chi^{2}$ minima and spectrum parameters determined.

\begin{tabular}{lcccr}
\hline \hline & \multicolumn{2}{c}{ Negative emission } & \multicolumn{2}{c}{ Bipolar emission } \\
& YBJ NM & SNT & YBJ NM & SNT \\
\hline$\chi^{2} /$ d.o.f. & $49.4 / 39$ & $46.2 / 39$ & $110 / 39$ & $59.7 / 39$ \\
$\alpha\left(\times 10^{3} \mathrm{MeV}^{-1} \mathrm{~m}^{-2} \mathrm{~s}^{-1}\right)$ a & $4.3 \pm 0.3$ & $4.2 \pm 0.3$ & $2.7 \pm 0.2$ & $2.8 \pm 0.2$ \\
$\left(\beta^{\mathrm{b}}, \mathrm{H}^{\mathrm{c}}\right)$ & \multicolumn{2}{c}{$(-2,0.9)$} & \multicolumn{2}{c}{$(-2,0.6)$} \\
\hline \hline
\end{tabular}

${ }^{\mathrm{a} A}$ A normalization factor of an assumed power-law gamma-ray spectrum.

${ }^{\mathrm{b}}$ An estimated photon index of a power-law gamma-ray spectrum.

${ }^{\mathrm{c}}$ A source height $(\mathrm{km})$ estimated. 

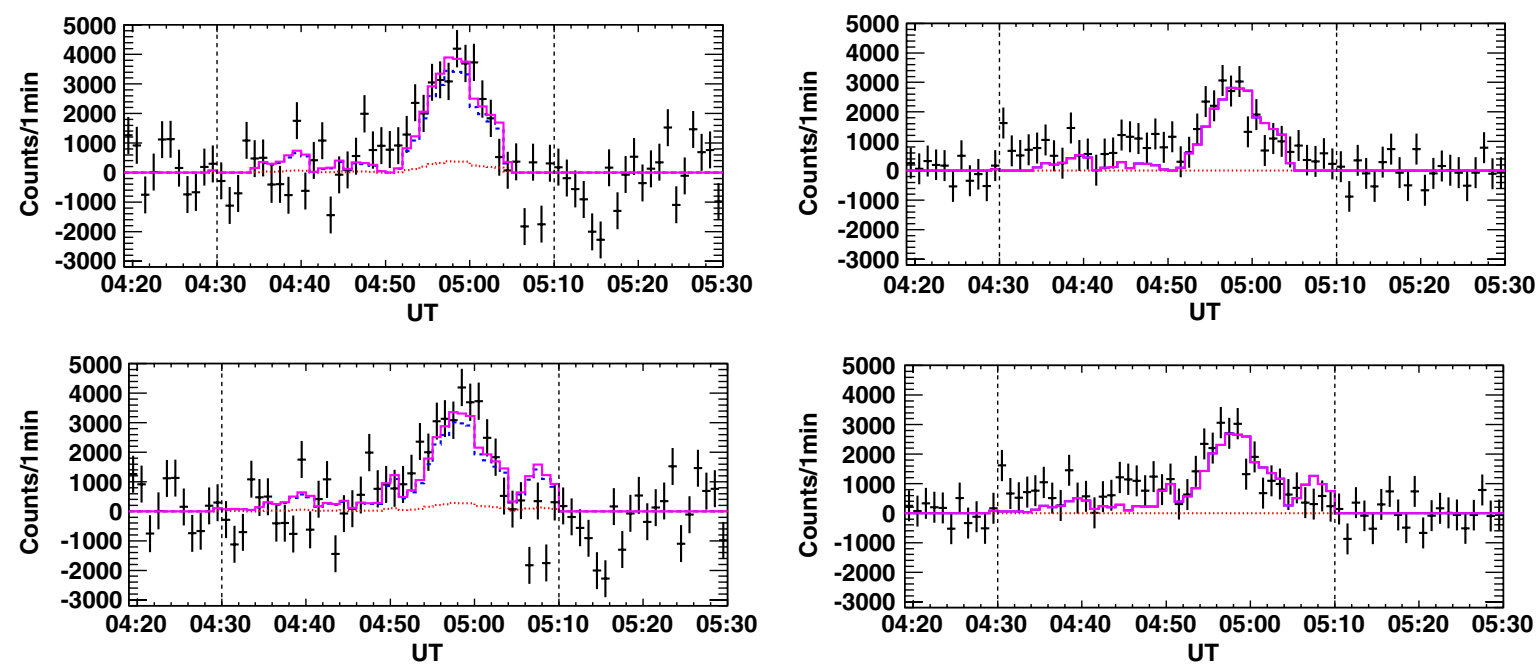

FIG. 11 (color online). Comparison of background-subtracted count histories with expected counts for 100722 . Left and right panels correspond to YBJ NM and the SNT ( $>40 \mathrm{MeV}$ ) with anticoincidence, respectively. Top and bottom panels represent the negative and bipolar emissions, respectively. Blue (dashed) and red (dotted) lines in each panel indicate a count history predicted by $\gamma$ rays and neutrons, respectively. Each magenta (solid) line shows an expected count history, which is summed over the counts from the relevant particles. Two vertical dashed lines in each panel denote the start and end times of the count increase.

minima, together with a set of $\beta$ and $H$. Figure $11 \mathrm{com}-$ pares the observed count histories of YBJ NM and the SNT with those expected from the parameters listed in Table II under the two emissions. The $\chi^{2}$ values (Table II) clearly suggest that the observed time profiles are reproduced by the negative emission rather than the bipolar emission.

\section{DISCUSSION}

\section{A. Gamma-ray emissions}

\section{Characteristics of $\gamma$-ray emissions}

The present study revealed that high-energy $\gamma$ rays with energies $>40 \mathrm{MeV}$ originate from summer thunderstorms. According to sea-level observations in winter thunderstorms [7], long-duration $\gamma$-ray emissions from winter thunderstorms extend to $10-20 \mathrm{MeV}$. This may be due to a difference in atmospheric density at ground and high mountains. In fact, a TGF spectrum on average extends to a few tens of $\mathrm{MeV}[38,39]$, or $100 \mathrm{MeV}$ on rare occasions [36]. It is believed that TGFs occur at altitudes of $15-20 \mathrm{~km}$ [40]. These results including the present one may imply that a lower atmospheric density is attributable to a higher $\gamma$-ray emission.

Compared with other thundercloud-related $\gamma$-ray events, the duration of the present event is exceptional with its long duration of $40 \mathrm{~min}$. Wash-out radioactive radon and its decay products frequently cause count increases in groundbased detectors. In addition, a duration of such a radon effect is around $20-30 \mathrm{~min}$ corresponding to their halflives. Thus, the duration of the radon effect is similar to the present one. However, the radon families generate $<3 \mathrm{MeV} \gamma$ rays, being unable to give signals in YBJ NM and the SNT.
According to electric-field measurement at the Tibet plateau (4.5 $\mathrm{km}$ a.s.l.) [29] and a mountain in New Mexico (3.2 km a.s.l.) [30], the mature stage of summer thunderclouds seems to last for $\sim 1 \mathrm{~h}$. In addition, the measurement in New Mexico revealed that a vertical potential relative to the surface in the mature stage is quasistable which is required for electrons to be continuously accelerated in thunderclouds in order to produce prolonged $\gamma$-ray emissions. Therefore, we infer that the present event is mainly associated with the mature stage of the Yangbajing thunderstorms. On the other hand, mature stages of winter thunderstorms at a costal area of Japan sea last for $<10 \mathrm{~min}$ [41]. In fact, all thundercloud-related $\gamma$ rays observed in winter lasted for at most a few minutes $[1,4,7,28]$. Thus, it is deduced that the longevity of the mature stage plays an important role in determining the duration of thundercloud-related $\gamma$ rays.

From the $\gamma$-ray emission of the present event, a source height $H$ was estimated as $H=900 \mathrm{~m}$ (Table I), giving the source altitude of $5.2 \mathrm{~km}$ a.s.l. Qie et al. [29] reported that a cloud base of summer thunderclouds above the Tibetan plateau (4.5 km a.s.1.) is generally located at $\sim 1 \mathrm{~km}$. In addition, Marshall et al. [42] clearly showed that a bottom positive layer of a summer thundercloud in New Mexico is located at $4.5-5.5 \mathrm{~km}$ a.s.l. Thus, the source altitude of $5.2 \mathrm{~km}$ a.s.l. is in good agreement with altitudes of the cloud base and the positive bottom layer obtained from these observations.

As clearly seen in Fig. 2, time structures for YBJ NM and the SNT are different from each other. In particular, YBJ NM showed no count increases at the burst onset, while all the SNT channels $(>40 \mathrm{MeV}$ to $>160 \mathrm{MeV}$ ) provided count enhancements in 5-10 min after the onset. 
These peculiar time structures might be caused by moving of thunderclouds and limited illumination of the higherenergy part of bremsstrahlung gamma rays emitted from thunderclouds. Actually, it is confirmed that long-duration gamma rays move with thunderclouds [7]. In addition, the bremsstrahlung gamma rays, especially gamma rays with an energy close to that of accelerated electrons, would be relativistically beamed into a narrow cone. For example, a half-opening angle of the cone, $\theta \sim 1 / \gamma$, is $0.1^{\circ}$ for a $300 \mathrm{MeV}$ electron, where $\gamma$ is a Lorentz factor. Given $H=$ $900 \mathrm{~m}$, we can obtain a radius of the gamma rays arriving at the observatory as at most $1.6 \mathrm{~m}\left(900 \mathrm{~m} \times \tan 0.1^{\circ}\right)$. Because YBJ NM is located $\sim 10 \mathrm{~m}$ apart from the $\mathrm{SNT},>40 \mathrm{MeV}$ gamma rays moving with thunderclouds might not happen to face toward YBJ NM during the burst onset.

\section{Electric potential}

Because of ionization loss of electrons, an electric potential of $40 \mathrm{MV}$ is not high enough to accelerate electrons to $40 \mathrm{MeV}$. In practice, an electric-field strength of $240-270 \mathrm{kV} \mathrm{m}^{-1}$ is required for electrons of $1-10 \mathrm{MeV}$ to be accelerated to $40 \mathrm{MeV}$ assuming a vertical length of a high-electric-field region is $0.5-1 \mathrm{~km}$, as determined by balloon experiments [42,43]. Multiplying this field strength by the assumed vertical length, the electric potential of at least $120 \mathrm{MV}$ must be established in the thunderclouds. This value of $120 \mathrm{MV}$ is approximately equal to the maximum potential of $130 \mathrm{MV}$ observed by balloon soundings [44]. In addition, the AGILE observation of TGFs showed that the electric potential in thunderstorms is on the order of $100 \mathrm{MV}$ [36] over macroscopic lengths such as cloud sizes or intracloud distances. Accordingly, the present observations may show manifestation of the highest potential field during thunderstorms.

\section{Avalanche multiplication factor}

In addition to quasistable electric fields, a stable or quasistable source of seed electrons would generally be needed for prolonged $\gamma$-ray emissions. Gurevich et al. [8] originally postulated that secondary cosmic rays consist of seed electrons, which increase in number and emit bremsstrahlung $\gamma$ rays. Thus, according to this premise, we derive an avalanche multiplication factor, $M$, expected from the RREA mechanism.

Using $\alpha$ and $\beta$ for the negative emission (Table II), a source $\gamma$-ray spectrum, $F_{\mathrm{s}}(E)$, can be described as $F_{\mathrm{s}}(E)=$ $\alpha_{\mathrm{w}} E^{-2}$. Here, $E$ is a photon energy in $\mathrm{MeV}$ and $\alpha_{\mathrm{w}}=$ $(4.3 \pm 0.2) \times 10^{3} \mathrm{~m}^{-2} \mathrm{~s}^{-1} \mathrm{MeV}^{-1}$ is a weighted mean calculated by the values of $\alpha$ from YBJ NM and the SNT. Using $F_{\mathrm{s}}$ and the burst duration $\Delta T=2400 \mathrm{~s}$, we estimated the total number of electrons with $10-100 \mathrm{MeV}$ energies as $N_{\mathrm{e}} \sim 10^{14}$, in the same manner as estimated by Tsuchiya et al. [7]. For this purpose we assumed a single acceleration region in the thundercloud with the vertical length and horizontal one of $Z=500 \mathrm{~m}$ or $1000 \mathrm{~m}$ and $L=600 \mathrm{~m}$ [7], respectively. In reality, a positive or a negative charge layer of thunderclouds may consist of multicells (e.g., [45]) to form several acceleration areas therein. Thus, the single acceleration region is a simple assumption to consider individual particle accelerations.

The secondary cosmic-ray electron flux above $1 \mathrm{MeV}$ at the relevant altitude is $I_{0} \sim 400 \mathrm{~m}^{-2} \mathrm{~s}^{-1}$ [46]. Therefore, the number of such electrons $N_{\text {cr }}$ entering the acceleration region in the burst period is computed as

$$
N_{\mathrm{cr}}=I_{0} \times A_{\mathrm{s}} \times \Delta T \sim 3 \times 10^{11}\left(\frac{L}{600 \mathrm{~m}}\right)^{2},
$$

giving $M$ as

$$
M=N_{\mathrm{e}} / N_{\text {cr }}=300\left(\frac{600 \mathrm{~m}}{L}\right)^{2} .
$$

Furthermore, based on the RREA mechanism, $M$ thus derived is described as

$$
M=\exp (Z / \lambda), \quad \lambda=\frac{7300 \mathrm{kV}}{V-276(P / 1 \mathrm{~atm})},
$$

where $\lambda$ and $V$ denote a length parameter given by Dwyer [10] and electric-field strength in $\mathrm{kV} \mathrm{m}^{-1}$, respectively. Substituting $M=300, Z=500$ or $1000 \mathrm{~m}$, and $P=$ $0.55 \mathrm{~atm}$ (average pressure at $H=900 \mathrm{~m}$ ) in Eq. (3), we obtain $V=240$ and $190 \mathrm{kV} \mathrm{m}^{-1}$ for $Z=500 \mathrm{~m}$ and $1000 \mathrm{~m}$, respectively. These values of $V$ are consistent with the above estimated field strength to accelerate electrons to $40 \mathrm{MeV}$ or higher energies.

Conducting sea-level observations in winter, Tsuchiya et al. [7] showed that secondary cosmic-ray electrons are multiplied by a factor of 3-30 to produce thundercloudrelated $\gamma$ rays. On the other hand, Chilingarian et al. [5] obtained a multiplication factor of $\sim 330$ with a highmountain measurement in summer. From these results as well as our result, a multiplication factor in high mountains can be considered to be different from that at sea level. However, the above $M$ becomes 30 if $L=2 \mathrm{~km}$, which is observed as the horizontal extent of a bottom positive layer in a summer thundercloud [42]. Thus, if $L$ is longer than $2 \mathrm{~km}$, the estimated $M$ may become consistent with that derived from sea-level observations.

\section{B. Neutron emissions}

\section{Comparison with the Aragats neutron monitor}

Similar to the present event, Chilingarian et al. [5] demonstrated that a count enhancement lasting for 10 min was detected by the Aragats neutron monitor located at $3250 \mathrm{~m}$ a.s.l. As a result, they concluded that the observed increase is fully attributable to neutrons related to the photonuclear reaction. On the other hand, the present results demonstrate that $>10 \mathrm{MeV} \gamma$ rays dominate the signals observed by YBJ NM. This is a 
main difference between the present study and that by Chilingarian et al. [5].

The present simulation clearly showed that an NM64 neutron monitor, which was also used by Chilingarian et al. [5], has low, but not negligible, sensitivity to $\gamma$ rays. In addition, the survival probability of neutrons and $\gamma$ rays at the Aragats observatory would not largely change from the present one (Fig. 8), because the air density at the Aragats observatory, which is $\sim 9 \times 10^{-4} \mathrm{~g} \mathrm{~cm}^{-3}$, is not very different from that at the Yangbajing site, which is $\sim 8 \times 10^{-4} \mathrm{~g} \mathrm{~cm}^{-3}$. In fact, using the GEANT4 simulation, Chilingarian et al. [5] derived $2.3 \times 10^{-3}$ as the survival probability of neutrons arriving at their observatory, assuming bremsstrahlung $\gamma$ rays propagate over $1500 \mathrm{~m}$. This value is nearly consistent with the survival probability of neutrons that is derived in the present study for $H=1500 \mathrm{~m}$, which is $5 \times 10^{-4}-2 \times 10^{-3}$ (Fig. 8). Consequently, not neutrons but $\gamma$ rays may possibly dominate enhancements detected by the Aragats neutron monitor.

\section{Number of neutrons produced}

Using the derived value of $\alpha_{\mathrm{w}}$, we evaluate the fluence of neutrons, $f_{\mathrm{n}}$, arriving at the observatory in energies $1 \mathrm{keV}-300 \mathrm{MeV}$, by the following formula:

$$
\begin{aligned}
f_{\mathrm{n}} & =\alpha_{\mathrm{w}} \Delta T \int_{\Delta T} I(t) d t \int_{1 \mathrm{keV}}^{300 \mathrm{MeV}} S_{\mathrm{n}}\left(E_{\mathrm{n}}\right) d E_{\mathrm{n}} \\
& =1.4 \times 10^{4} \mathrm{~m}^{-2},
\end{aligned}
$$

where $\Delta T=2400 \mathrm{~s}$ and $S_{\mathrm{n}}\left(E_{\mathrm{n}}\right)$ represents the simulated neutron spectrum, assuming $\beta$ and $H$ are -2 and $900 \mathrm{~m}$ (Table II), respectively. Carlson et al. [17] and Babich et al. [18] described that photonuclear neutrons produced by energetic $\gamma$ rays are observable at ground level when a $\gamma$-ray source is locate $<5 \mathrm{~km}$, since the neutron fluence is expected as $(0.03-1) \times 10^{4} \mathrm{~m}^{-2}$ for the former prediction and $10^{3}-10^{7} \mathrm{~m}^{-2}$ for the latter one. Actually, the value of $f_{\mathrm{n}}$ is consistent with their predictions. Thus, this agreement implies that the photonuclear reaction certainly occurs during mature stages of thunderclouds.

\section{SUMMARY}

The prolonged $\gamma$-ray event, lasting for $40 \mathrm{~min}$, was observed on July 22, 2010, at Yangbajing in Tibet, China. Such a long-duration event associated with thunderstorms has never been observed. In addition, the present observations clearly showed that $\gamma$ rays extending to energies $>40 \mathrm{MeV}$ were detected by the SNT and very likely by YBJ NM. Given these results, the present emissions strongly suggest that electrons are accelerated beyond at least $40 \mathrm{MeV}$ in $40 \mathrm{~min}$, by quasistable electric fields, which were formed during the mature stage of summer thunderclouds. The present duration is at least 5 times longer than those observed in winter thunderstorms at the coastal area of the Japan sea. Probably, one of the main reasons for this difference would be ascribed to a difference in life cycles of mature stages of winter and summer thunderclouds.

The high-energy $\gamma$ rays would produce neutrons via the photonuclear reaction of ${ }^{14} \mathrm{~N}(\gamma, n){ }^{13} \mathrm{~N}$. The present simulation showed that the arriving neutron flux at $>1 \mathrm{keV}$ is expected to be lower than that of arriving $\gamma$ rays at $>10 \mathrm{MeV}$ by more than 2 orders of magnitude. Moreover, it revealed that unlike previously believed, neutron monitors are not insensitive to $\gamma$ rays. Consequently, it is found that bremsstrahlung $\gamma$ rays largely attribute the signal obtained by YBJ NM and photonuclear neutrons give only a small contribution to the signal. The present study demonstrated that worldwide networks of neutron monitors [27] and solar-neutron telescopes [47,48] are useful for observations of thunderstorm-related $\gamma$-ray emissions.

\section{ACKNOWLEDGMENTS}

The study is supported in part by a Grant-in-Aid for Scientific Research (C), No. 20540298, and a Grantin-Aid for Young Scientists (B), No. 19740167. This study is also supported in part by the Special Postdoctoral Research Project for Basic Science in RIKEN, the Special Research Project for Basic Science in RIKEN ("Investigation of Spontaneously Evolving Systems").
[1] H. Tsuchiya et al., Phys. Rev. Lett. 99, 165002 (2007).

[2] H. Tsuchiya et al., Phys. Rev. Lett. 102, 255003 (2009).

[3] T. Torii, T. Sugita, S. Tanabe, Y. Kimura, M. Kamogawa, K. Yajima, and H. Yasuda, Geophys. Res. Lett. 36, L13804 (2009).

[4] T. Torii, T. Sugita, M. Kamogawa, Y. Watanabe, and K. Kusunoki, Geophys. Res. Lett. 38, L24801 (2011).

[5] A. Chilingarian, A. Daryan, K. Arakelyan, A. Hovhannisyan, B. Mailyan, L. Melkumyan, G.
Hovsepyan, S. Chilingaryan, A. Reymers, and L. Vanyan, Phys. Rev. D 82, 043009 (2010).

[6] A. Chilingarian, G. Hovsepyan, and A. Hovhannisyan, Phys. Rev. D 83, 062001 (2011).

[7] H. Tsuchiya et al., J. Geophys. Res. 116, D09113 (2011).

[8] A. V. Gurevich, G. M. Milikh, and R. Roussel-Dupre, Phys. Lett. A 165, 463 (1992).

[9] A. V. Gurevich and G. M. Milikh, Phys. Lett. A 262, 457 (1999).

[10] J. R. Dwyer, Geophys. Res. Lett. 30, 2055 (2003). 
[11] V. V. Alexeenko, N. S. Khaerdinov, A. S. Lidvansky, and V. B. Petkov, Phys. Lett. A 301, 299 (2002).

[12] Y. Muraki et al., Phys. Rev. D 69, 123010 (2004).

[13] G. N. Shah, H. Razdan, C. L. Bhat, and Q. M. Ali, Nature (London) 313, 773 (1985).

[14] A. Shyam and T. C. Kaushik, J. Geophys. Res. 104, 6867 (1999).

[15] B. L. Babich and R. A. Roussel-Dupré, J. Geophys. Res. 112, D13303 (2007).

[16] B. L. Berman, At. Data Nucl. Data Tables 15, 319 (1975).

[17] B. E. Carlson, N. G. Lehtinen, and U. S. Inan, J. Geophys. Res. 115, A00E19 (2010).

[18] L.P. Babich, E. I. Bochkov, E. N. Donskoi, and I. M. Kutsyki, J. Geophys. Res. 115, A09317 (2010).

[19] M. Amenomori et al., Astrophys. J. 692, 61 (2009).

[20] H. Tsuchiya et al., Astron. Astrophys. 468, 1089 (2007).

[21] Y. Muraki et al., Astropart. Phys. 28, 119 (2007).

[22] H. Carmichael, Cosmic Rays, IQSY Instruction Manual No. 7 (IQSY Secretariat, London, 1964).

[23] P. H. Stoker, L. I. Dorman, and J. M. Clem, Space Sci. Rev. 93, 361 (2000).

[24] S. Agostinelli et al., Nucl. Instrum. Methods Phys. Res., Sect. A 506, 250 (2003).

[25] J. M. Clem and L. I. Dorman, Space Sci. Rev. 93, 335 (2000).

[26] S. Shibata et al., Nucl. Instrum. Methods Phys. Res., Sect. A 463, 316 (2001).

[27] I. G. Usoskin, G. A. Kovaltsov, H. Kananen, and P. Tanskanen, Ann. Geophys. 15, 375 (1997).

[28] T. Torii, M. Takeishi, and T. Hosono, J. Geophys. Res. 107, 4324 (2002).

[29] X. Qie, T. Zhang, C. Chen, G. Zhang, T. Zhang, and W. Wei, Geophys. Res. Lett. 32, L05814 (2005).

[30] M. Stolzenburg and T. C. Marshall, J. Geophys. Res. 113, D13207 (2008).

[31] L.P. Babich, E. I. Bochkov, I. M. Kutsyk, and R. A. Roussel-Dupré, J. Geophys. Res. 115, A00E28 (2010).
[32] S. Shibata, J. Geophys. Res. 99, 6651 (1994).

[33] S. Hakmana Witharana, Ph.D. thesis, Georgia State University, 2007.

[34] J. R. Dwyer, Geophys. Res. Lett. 31, L12102 (2004).

[35] L. P. Babich, E. N. Donskoy, R. I. Il'kaev, I. M. Kutsyk, and R.A. Roussel-Dupre, Plasma Phys. Rep. 30, 616 (2004).

[36] M. Tavani et al., Phys. Rev. Lett. 106, 018501 (2011).

[37] V. A. Rakov and M.A. Uman, Lightning Physics and Effects (Cambridge University Press, Cambridge, England, 2005), p. 67.

[38] M. Marisaldi et al., J. Geophys. Res. 115, A00E13 (2010).

[39] M.S. Briggs et al., J. Geophys. Res. 115, A07323 (2010).

[40] J. R. Dwyer and D. M. Smith, Geophys. Res. Lett. 32, L22804 (2005).

[41] N. Kitagawa and K. Michimoto, J. Geophys. Res. 99, 10713 (1994).

[42] T. C. Marshall, M. Stolzenburg, C. R. Maggio, L. M. Coleman, P. R. Krehbiel, T. Hamlin, R. J. Thomas, and W. Rison, Geophys. Res. Lett. 32, L03813 (2005).

[43] K. B. Eack, W. H. Beasley, W. D. Rust, T. C. Marshall, and M. Stolzenburg, J. Geophys. Res. 101, 29637 (1996).

[44] T. C. Marshall and M. Stolzenburg, J. Geophys. Res. 106, 4757 (2001).

[45] W. W. Hager, B. C. Aslan, R. G. Richard, G. Sonnenfeld, T. D. Crum, J. D. Battles, M. T. Holborn, and R. Ron, J. Geophys. Res. 115, D12119 (2010).

[46] P. K. F. Grieder, Cosmic Rays at Earth (Elsevier Science B. V., Amsterdam, 2001), p. 198.

[47] H. Tsuchiya et al., Nucl. Instrum. Methods Phys. Res., Sect. A 463, 183 (2001).

[48] Y. Matsubara et al., Proc. 30th Int. Cosmic Ray Conf. (Universidad Nacional Autonoma de Mexico, Mexico City, 2007), Vol. 1, p. 29. 